\title{
ORLICZ SPACES FOR WHICH THE HARDY-LITTLEWOOD MAXIMAL OPERATOR IS BOUNDED
}

\author{
DIEGO GALLARDO
}

Abstract

Let $M$ be the Hardy-Littlewood maximal operator defined by

$$
M f(x)=\sup _{x \in Q} \frac{1}{|Q|} \int_{Q}|f| d x \quad, \quad\left(f \in L_{\operatorname{loc}}\left(\mathbb{R}^{n}\right)\right)
$$

where the supremum is taken over all cubes $Q$ containing $x$ and $|Q|$ is the Lebesgue measure of $Q$. In this paper we characterize the Orlicz spaces $L_{\phi}^{*}$, associated to $N$-functions $\phi$, such that $M$ is bounded in $L_{\phi}^{*}$. We prove that this boundedness is equivalent to the complementary $N$-function $\psi$ of $\phi$ satisfying the $\Delta_{2}$-condition in $[0, \infty)$, that is, $\sup _{s}>0 \psi(2 s) / \psi(s)<\infty$.

\section{Introduction}

It is known that for the Hardy-Littlewood maximal operator, on $\mathrm{R}$, defined by

$$
\theta(t ; f)=\sup _{|t-\tau|>0} \frac{1}{\tau-t} \int_{t}^{\tau}|f(s)| d s
$$

to act boundedly in a symmetric space $E$ it is necessary and sufficient that the following condition be satisfied

$$
\left\|\sigma_{\tau}\right\|_{E}=o(\tau) \text { as } \tau \rightarrow \infty,
$$

where $\sigma_{\tau}$ are the dilation operators defined by $\sigma_{\tau} f(t)=f\left(\tau^{-1} t\right)$. (See Ch. II, Theorem 6.10 in [2]).

In this paper we consider the more general Hardy-Irittlewood maximal operator $M$, on $R^{n}$, defined by

$$
M f(x)=\sup _{x \in Q} \frac{1}{|Q|} \int_{Q}|f| d x \quad, \quad\left(f \in L_{\mathrm{loc}}^{1}\left(\mathrm{R}^{n}\right)\right),
$$

This research has been supported by a CAYCIT Grant (PB85-0434). 
where the supremum is taken over all cubes $Q$ containing $x$ and $|Q|$ is the Lebesgue measure of $Q$. (Cube will mean a compact cubic interval with nonempty interior).

Our aim is to characterize the Orlicz spaces $L_{\phi}^{*}$, associated to $N$-functions $\phi$, for which the operator defined by (1.3) is bounded. Such Orlicz spaces are symmetric spaces, but, for the case $n=1$, the proof given in here represents a direct and different proof from that given in [2]. Moreover, our characterizing condition is more manageable than (1.2).

Except in basic questions, this paper is intended to be selfcontained.

Now, we shall present the basic definitions and results concerning to $\mathrm{N}$ functions and Orlicz spaces which will be used in this paper. The proofs of most of these results can be found in [1] or in II-13 of [3].

An $N$-function is a continuous and convex function $\phi:[0, \infty) \rightarrow R$ such that $\phi(s)>0, s>0, s^{-1} \phi(s) \rightarrow 0$, as $s \rightarrow 0$, and $s^{-1} \phi(s) \rightarrow \infty$, as $s \rightarrow \infty$.

As example of $N$-functions we have: $\phi_{1}(s)=s^{p}, p>1 ; \phi_{2}(s)=s^{p} \log ^{k}(1+s)$, $p \geq 1$ and $k>0 ; \phi_{4}(s)=e^{s}-s-1 ; \phi_{5}(s)=(1+s) \log (1+s)-s ; \phi_{5}(s)=$ $=\exp s^{2}-1$ and $\phi_{6}(s)=\int_{0}^{s} \rho$ where $\rho:[0, \infty) \rightarrow[0, \infty)$ is defined by $\rho(0)=0$, $\rho(t)=2^{-n}$ if $t \in\left[2^{-n}, 2^{-n+1}\right)$ and $\rho(t)=2^{n-1}$ if $t \in\left[2^{n-1}, 2^{n}\right), n$ a positive integer.

An $N$-function $\phi$ has the representation $\phi(s)=\int_{0}^{s} \varphi$ where $\varphi:[0, \infty) \rightarrow R$ is continuous from the right, non decreasing such that $\varphi(s)>0, s>0, \varphi(0)=0$ and $\varphi(s) \rightarrow \infty$ for $s \rightarrow \infty$. More precisely $\varphi$ is the right derivate of $\phi$ and will be called the density function of $\phi$.

Associated to $\varphi$ we have the function $\rho:[0, \infty) \rightarrow \mathbf{R}$ defined by $\rho(t)=\sup \{s$ : $\varphi(s) \leq t\}$ which has the same aforementioned properties of $\varphi$. We will call $\rho$ the generalized inverse of $\phi$.

The $N$-function $\psi$ defined by $\psi(t)=\int_{0}^{t} \rho$ is called the complementary $N-$ function of $\phi$. Thus, if $\phi(s)=p^{-1} s^{p}, p>1$, then $\psi(t)=q^{-1} t^{q}$ where $p q=p+q$.

Young's inequality asserts that st $\leq \phi(s)+\psi(t)$ for $s, t \geq 0$, equality holding if and only if $\varphi(s-) \leq t \leq \varphi(s)$ or else $\rho(t-) \leq s \leq \rho(t)$.

An $N$-function $\phi$ is said to satisfy the $\Delta_{2}$-condition in $[0, \infty$ ) (or merely the $\Delta_{2}$-condition) if $\sup _{9}>0 \phi(2 s) / \phi(s)<\infty$. If $\varphi$ is the density function of $\phi$, then, $\phi$ satisfies $\Delta_{2}$ if and only if there exists a constant $\alpha>1$ such that $s \varphi(s)<\alpha \phi(s), s>0$.

The $\Delta_{2}$-condition for $\phi$ does not transfer necessarily to the complementary $N$-function; for example, $\phi$ defined by $\phi(s)=(1+s) \log (1+s)-s$ satisfies the $\Delta_{2}$-condition but its complementary $N$-function $\psi$, defined by $\psi(t)=e^{t}-t-1$, does not. In this paper the $\Delta_{2}$-condition for the complementary $N$-function $\psi$ of $\phi$ plays a fundamental role; precisely, this is the characterizing condition for the boundeddness of the Hardy-Littlewood maximal operator defined in (1.3). For this reason it is very interesting to give some characterizations of this condition, which permit to know wheter $\psi$ satisfies $\Delta_{2}$ even if we do not 
know explicitly the function $\psi$.

First, it is known that $\psi$ satisfies the $\Delta_{2}$-condition in $[0, \infty)$ if and only if there exists a constant $a>1$ such that $\phi(s) \leq(2 a)^{-1} \phi(a s), s \geq 0$.

The following characterization, which is used in this paper, appear in the literature with more restrictive hypothesis than the one we shall use and so we shall include its proof.

Proposition 1.4. The complementary $N$-function of $\phi$ satisfies the $\Delta_{2}-$ condition in $[0, \infty)$ if and only if inf $f_{s}>0 s \varphi(s) / \phi(s)>1$, where $\varphi$ is the density function of $\phi$.

Proof of (1.4): Let $\rho$ be the generalized-inverse of $\varphi$ and let $\psi$ be the complementary $N$-function of $\phi$. Assume that $\psi$ satisfies $\Delta_{2}$; then, there exists $\alpha>1$ such that $t \rho(t)<\alpha \psi(t)$ for every $t>0$. On the other hand, it follows from the equality cases in Youngs's inequality that $t \rho(t)=\phi(\rho(t))+\psi(t)$ and therefore $\phi(\rho(\varphi(s))) / \rho(\varphi(s))<\alpha^{-1}(\alpha-1) \varphi(s), s>0$. Since $\rho(\varphi(s)) \geq s$ and the function $u \rightarrow u^{-1} \phi(u)$ increases strictly for $u>0$ we obtain that $\inf _{s>0} s \varphi(s) / \phi(s)>\alpha(\alpha-1)^{-1}$.

Assume now that $\inf _{s>0} s \varphi(s) / \phi(s)>1$; then, there exists $\beta>1$ such that the function $s \rightarrow s^{-\beta} \phi(s)$ increases strictly for $s>0$ and, therefore, there exists $a>1$ such that $\phi(s)<(2 a)^{-1} \phi(a s), s>0$. Thus, $\psi$ satisfies $\Delta_{2}$ and this finishes the proof.

If $\left(X, \mathcal{M}_{1} \mu\right)$ is a $\sigma$-finite measure space we denote by $\mathfrak{M}$ the space of $\mathcal{M}$ measurable and $\mu$-a.e. finite functions from $X$ to $B$ (or to $C$ ). If $\phi$ is an $N$-function the Orlicz spaces $L_{\phi} \equiv L_{\phi}(X, \mathcal{M}, \mu)$ and $L_{\phi}^{*} \equiv L_{\phi}^{*}(X, \mathcal{M}, \mu)$ arc defined by $L_{\phi}=\left\{f \in \mathfrak{M}: \int_{X} \phi(|f|) d \mu<\infty\right\}$ and $L_{\phi}^{*}=\left\{f \in \mathfrak{M}: f g \in L_{1}(\mu)\right.$ for all $\left.g \in L_{\psi}\right\}$, where $\psi$ is the complementary $N$-function of $\phi$.

We have $L_{\phi} \subset L_{\phi}^{*}$ and $L_{\phi}^{*}$ coincides with the set of $\mathfrak{M}$ such that $\lambda f \in L_{\phi}$ for some positive real $\lambda$.

The space $L_{\phi}^{*}$ is a linear space with the usual operations and we may define the following norms in $L_{\phi}^{*}$ :

$$
\|f\|_{\phi}=\sup \left\{\int_{X}|f g| d \mu: g \in S_{\psi}\right\}
$$

where $S_{\psi}=\left\{g \in L_{\psi}: \int_{X} \psi(|g|) d \mu \leq 1\right\}$, and

$$
\|f\|_{\phi)}=\inf \left\{\lambda>0: \int_{X} \phi\left(\lambda^{-1}|f|\right) d \mu \leq 1\right\},
$$

which are called the Orlicz norm and the Luxemburg norm respectively. Both norms are equivalent, actually $\|f\|_{(\phi)} \leq\|f\|_{\phi} \leq 2\|f\|_{(\phi)}$, and they make $L_{\phi}^{*}$ into a Banach space. 
For a measurable set, $A$, with $0<\mu(A)<\infty,\left\|\chi_{A}\right\|_{\phi}=\mu(A) \psi^{-1}(1 / \mu(A))$, where $\chi_{A}$ denotes the characteristic function of $A$.

If $\phi(s)=s^{p}, p>1$, then, $L_{\phi}^{*}=L_{\phi}=L_{p},\|f\|_{(\phi)}=\|f\|_{p}$ and $\|g\|_{\psi}=\|g\|_{q}$, where $p q=p+q$.

\section{The main result}

Theorem 2.1. Let $\phi$ an $N$-function, $\psi$ the complementary $N$-function of $\phi$, $L_{\phi}^{*}$ the Orlicz space associated to $\phi$ and let $M$ be the Hardy-Littlewood maximal operator defined in (1.8). The following conditions are equivalent:

(a) There exist positive constants $A$ and $b$ such that

$$
\int_{\mathbf{R}^{n}} \phi(b M f) d x \leq A \int_{\mathbf{R}^{n}} \phi(|f|) d x \quad, \quad\left(f \in L_{\phi}^{*}\right) .
$$

(b) There exists a positive constant $C$ such that

$$
\|M f\|_{(\phi)} \leq C\|f\|_{(\phi)} \quad, \quad\left(f \in L_{\phi}^{*}\right) .
$$

(c) There exists a positive constant $K$ such that

$$
\left\|M_{\chi_{A}}\right\|_{(\phi)} \leq K^{\prime}\left\|\chi_{A}\right\|_{(\phi)} \quad, \quad(|A|<\infty) .
$$

(d) $\psi$ satisfies the $\Delta_{2}$-condition in $[0, \infty)$.

Proof: It suffices to prove $(a) \Rightarrow(b),(c) \Rightarrow(d)$ and $(d) \Rightarrow(a)$.

The proof of $(a) \Rightarrow(b)$ is easy. In fact, if $f \in L_{\phi}^{*}$ there exists $\lambda>0$ such that $\lambda f \in L_{\phi}$, hence $\lambda b M f \in L_{\phi}$ and therefore $M f \in L_{\phi}^{*}$. Moreover, we have

$$
\begin{aligned}
& \int_{\mathbb{R}^{n}} \phi\left(M f /\left(b^{-1} \max (1, A)\|f\|_{(\phi)}\right)\right) d x< \\
& <A(\max (1, A))^{-1} \int_{\mathbf{R}^{n}} \phi\left(|f| /\|f\|_{(\phi)}\right) d x \leq 1,
\end{aligned}
$$

for $f \neq 0$ and thus we get $\|M f\|_{(\phi)} \leq b^{-1} \max (1, A)\|f\|_{(\phi)}$ for every $f \in L_{\phi}^{*}$.

The proof of $(d) \Rightarrow(a)$ follows from the following interpolation result, taking into account that $M$ is of weak type $(1,1)$ and bounded in $L_{\infty}$.

Theorem 2.2. Let $(X, \mathcal{M}, \mu)$ and $(Y, \mathcal{F}, v)$ be two $\sigma$-finite measure spaces, $\phi$ an $N$-function whose complementary $N$-function satisfies the $\Delta_{2}$-condition and let $T: L_{1}(\mu)+L_{\infty}(\mu) \rightarrow \mathfrak{M}(Y)$ be a quasi-additive operator which is simultaneously of weak type $(1,1)$ and of type $(\infty, \infty)$. Then, $T$ is defined on $L_{\phi}^{*}(\mu)$ and there exist positive constants $A$ and $m$ such that

$$
\int_{Y} \phi(|T f|) d v \leq A \int_{X} \phi(m|f|) d \mu \quad, \quad\left(f \in L_{\phi}^{*}(\mu)\right) .
$$


Proof of Theorem 2.2: By hypothesis there exists a constant $C$ such that

$$
\begin{aligned}
& v\{y \in Y:|T g(y)|>\lambda\} \leq C \lambda^{-1} \int_{X}|g| d \mu, \\
& \|T h\|_{\infty} \leq C|| h \|_{\infty}, \quad|T(g+h)| \leq C(|T g|+|T h|)
\end{aligned}
$$

for every $g \in L_{1}(\mu), h \in L_{\infty}(\mu)$ and $\lambda>0$.

For $f \in L_{\phi}^{*}$ and $\lambda>0$ let $f_{\lambda}=f \chi_{A\left(\lambda / 2 C^{2}\right)}$ and $f^{\lambda}=f-f_{\lambda}$ where $A(\alpha)=$ $=\{x \in X:|f(x)|>\alpha\}$. We have $f_{\lambda} \in L_{1}(\mu)$ and $f^{\lambda} \in L_{\infty}(\mu)$ and therefore

$$
\begin{aligned}
& \int_{Y} \phi(|T f|) d v=\int_{0}^{\infty} \varphi(\lambda) v\{y \in Y:|T f(y)|>\lambda\} d \lambda \leq \\
& \leq 2 C^{2} \int_{0}^{\infty} \lambda^{-1} \varphi(\lambda)\left(\int_{X}\left|f_{\lambda}\right| d \mu\right) d \lambda= \\
& =2 C^{2} \int_{X}|f(x)|\left(\int_{0}^{2 C^{2}|f(x)|} \lambda^{-1} \varphi(\lambda) d \lambda\right) d \mu(x),
\end{aligned}
$$

where $\varphi$ is the density function of $\phi$.

Integrating by parts, we obtain

$$
\int_{0}^{s} \lambda^{-1} \varphi(\lambda) d \lambda=s^{-1} \phi(s)+\int_{0}^{s} \lambda^{-2} \phi(\lambda) d \lambda, \quad(s>0) .
$$

Since $\psi$ satisfies $\Delta_{2}$, it follows from Proposotion 1.4 that there exists $\beta>1$ such that $\beta \phi(s)<s \varphi(s), s>0$; then, if $0<\lambda<1$ we have that $\lambda^{-2} \phi(\lambda) \leq$ $\phi(1) \lambda^{\beta-2}$ and therefore $\int_{0}^{3} \lambda^{-2} \phi(\lambda) d \lambda$ is finite. then (2.4) shows that

$$
\int_{0}^{s} \lambda^{-1} \varphi(\lambda) d \lambda<\beta(\beta-1)^{-1} s^{-1} \phi(s) \quad, \quad(s>0)
$$

and thus, we obtain (2.3) with $A=\beta(\beta-1)^{-1}$ and $m=2 C^{2}$.

Note. If $T$ is also positively homogenous then it follows from (2.3) that

$$
\int_{Y} \phi\left(m^{-1}|T f|\right) d v \leq A \int_{X} \phi(|f|) d \mu \quad, \quad\left(f \in L_{\phi}^{*}(\mu)\right)
$$

and $T$ applies $L_{\phi}^{*}(X, \mathcal{M}, \mu)$ in $L_{\phi}^{*}(Y, \mathcal{F}, v)$.

Proof of $(c) \Rightarrow(d)$ : It follows from (c) that there exists a constant $K>0$ such that $\left\|M_{E} \chi_{A}\right\|_{\phi} \leq K\left\|\chi_{A}\right\|_{\phi}$ for every $A$ with $|A|<\infty$, with $M_{E}$ being the maximal operator on balls, defined by $M_{E} f(x)=\sup _{x \in B}|B|^{-1} f_{B}|f| d x$, where the supremum is taken over all euclideans balls $B$ containing $x$ and \|\|$_{\phi}$ is the Orlicz norm.

We denote by $B(x ; r)$ the ball with center $x$ and radius $r$ and let $a_{n}$ be the measure of $B(0 ; 1)$. For every pair of reals $(v, s)$, with $v>0$ and $s>1$, we 
denote by $A(v, s)$ and $D(v, s)$ the balls $B\left(0 ;\left(a_{n} v s\right)^{-1 / n}\right)$ and $B\left(0 ;\left(a_{n} v\right)^{-1 / n}\right)$ respectively.

If $x \notin A(v, s)$ then $A(v, s) \subset B\left(x ; 2\|x\|_{2}\right)$ and therefore

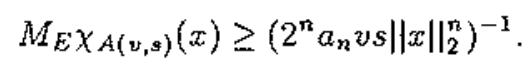

On the other hand, if $g=\psi^{-1}(v) \chi D(v, s)$ we have $\int_{\mathbf{R}^{n}} \psi(|g|) d x=1$ and consequently

$$
\begin{aligned}
& \left\|M_{E \chi A(v, s)}\right\|_{\phi} \geq \psi^{-1}(v) \int_{D(v, s)} M_{E \chi_{A(v, s)}} d x \geq \\
& \geq\left(2^{n} a_{n} v s\right)^{-1} \psi^{-1}(v) \int_{\left(a_{n} v s\right)^{-1 / n}<\|x\|_{2}<\left(a_{n} v\right)^{-1 / n}}\|x\|_{2}^{-n} d x= \\
& =\left(2^{n} v s\right)^{-1} \psi^{-1}(v) \log s .
\end{aligned}
$$

Since $\left\|\chi_{A(v, s)}\right\|_{\phi}=(v s)^{-1} \psi^{-1}(v s)$ we conclude that there exists $K>0$ such that

$$
2^{-n} \psi^{-1}(v) \log s \leq K \psi^{-1}(s v) \quad(v>0)(s>1) .
$$

Therefore $2 \psi^{-1}(v) \leq \psi^{-1}\left(v \exp \left(K 2^{n+1}\right)\right), v>0$, which proves that $\psi(2 t) \leq$ $\leq \exp \left(K 2^{n+1}\right) \psi(t)$ for every $t>0$ and so $\psi$ satisfies $\Delta_{2}$ in $[0, \infty)$.

Final Remark. It follows from Theorem 2.1 that the $\Delta_{2}-$ condition on the complementary $N$-function of $\phi$ cannot be eliminated from the hypothesis of the interpolation theorem 2.2 since if the complementary $N$-function of $\phi$ does not satisfy $\Delta_{2}$ (for example, in the case of $\phi$ defined by $\phi(s)=(1+s) \log (1+$ $+s)-s$ ) the result of the interpolation theorem does not hold for $\phi$ and the Hardy-Littlewood maximal operator in spitc of the fact that this operator is of weak type $(1,1)$ and of type $(\infty, \infty)$. (Moreover, observe that in the abovementioned case the $N$-function $\phi$ satisfies the $\Delta_{2}$-condition).

\section{References}

1. M.A. KRasnoselsky and V.B. Rutitsky, Convex functions and Orlicz spaces, Noordhoff Groningen (1961).

2. S.G. KREIN, J.I. PETUNin AND E.M. SEmenov, Interpolation of Linear Operators, Translations of Mathematical Monographs (1982), Amer. Math. Soc.

3. J. MusielaK, "Orlicz spaces and modular spaces," Springer-Verlag, 1983.

Departamerito de Matemáticas

Facultad de Ciencias

Universidad de Málaga, SPAIN. 